\title{
MOSP: Monitor of Outer Scale profile
}

\author{
Aziz Ziad ${ }^{1, a}$, Jérôme Maire ${ }^{2}$, Julien Borgnino ${ }^{1}$, Wassila Dali Ali ${ }^{1}$, Amokrane Berdja ${ }^{1}$, Khaled Ben \\ Abdallah $^{1}$, François Martin ${ }^{1}$, and Marc Sarazin \\ 1 UMR 6525 H. Fizeau, Université de Nice Sophia-Antipolis, CNRS, Observatoire de la Côte d'Azur, \\ Parc Valrose 06108, Nice, France \\ 2 Centre de Recherche en Astrophysique du Québec, Département de Physique, Université de Montréal \\ C.P. 6128, Qc, H3C 3J7, Montréal, Canada
}

\begin{abstract}
The outer scale profile is a key parameter for the specification and optimization of the Adaptive Optics systems for the Extremely Large Telescopes. A new instrument MOSP for $\mathcal{L}_{0}(h)$ extraction has been developed and several campaigns have been carried out at Mauna Kea, Paranal and now at Dome C in Antarctica. The MOSP instrument and results obtained during his observation campaigns are presented and discussed.
\end{abstract}

\section{Introduction}

Wavefront outer scale is a relevant parameter for the experimental performance evaluation of large aperture telescopes. The actual size of the outer scale has long been controversial, with measured values ranging from less than $10 \mathrm{~m}$ to more than $2 \mathrm{~km}$. What is not controversial is the conclusion that when the diameter of the telescope approaches or exceeds the size of the outer scale, the optical consequences of atmospheric turbulence are changed dramatically from their traditional Kolmogorov behavior. In particular, power in the lowest Zernike aberration modes, e.g., tip and tilt and the overall stroke required for an adaptive-optics system can be much reduced. A finite outer scale has implications for interferometry as well. With the current interest in the design of extremely large ground-based optical and infrared telescopes, reliable estimates of the outer scale profile have assumed considerable importance. A new instrument MOSP (Monitor of Outer Scale Profile) has been developed by our team for outer scale profile extraction. We retrieve the vertical distribution of wavefront outer scale by analysing angular correlation of wavefront Angle of Arrival fluctuations deduced from Moon's limb images motion. We use simulated annealing algorithm to deduce the height dependence of the wavefront outer scale with given $C_{N}^{2}$ profiles simultaneously measured with the SCIDAR instrument. We present results obtained during three campaigns of observation at the Mauna Kea Observatory (Hawaii) at the Paranal VLT Observatory (Chile) and at the Observatoire de Haute Provence (France). Estimated outer scale profiles exhibit smaller values in the boundary layer than in the free atmosphere. Comparisons with GSM outer scale measurements are possible and give good agreement. Some implications for adaptive optics systems are considered.

\section{MOSP instrument}

\subsection{Description}

The principle of the MOSP instrument is based on the angular correlation of wavefront Angle of Arrival (AA) fluctuations deduced from Moon's limb image motion. The AA fluctuations are measured perpendicularly to the lunar limb leading to transverse correlations for different angular separation along the moon.

The MOSP instrument consists of small telescope $(D \leq 60 \mathrm{~cm})$ having a large focal length $f \sim 10 \mathrm{~m}$. In the case a telescope with small $f$, a diverging Barlow lens is used increasing the effective focal

\footnotetext{
a e-mail: ziad@unice.fr
} the original work is properly cited. 


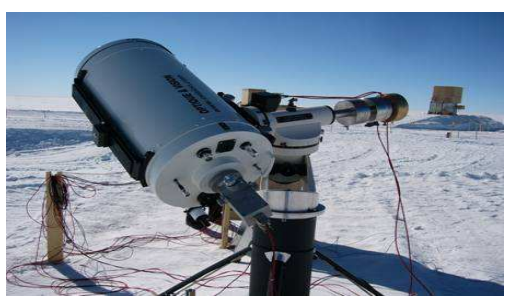

Fig. 1. MOSP at the Dome $\mathrm{C}$ site in Antarctica.

length of the system, thereby increasing the magnification of the instrument. But this is to the detriment to the field of view (FOV). A compromise has to be found between these parameters.

Images at the focal plane are recorded using a PixelFly CCD camera with $640 \times 480$ pixel matrix and $(9.9 \times 9.9) \mu^{2}$ pixel size. Its dynamic range of the analogic/digital conversion is 12 bits. The readout noise is $12 \mathrm{e}-\mathrm{rms}$ and the imaging frequency is $33 \mathrm{~Hz}$. In order to freeze atmospheric effects on Moon's limb image motion, the exposure time was set to 1 or $2 \mathrm{~ms}$. The spectral response of the camera is maximal for $\lambda=0.5 \mu \mathrm{m}$ in a $375-550 \mu \mathrm{m}$ range.

\subsection{Data processing}

The first step of data processing is to retrieve accurately AA fluctuations from Moon's limb motion. After processing on each image a flat and dark field correction, each image $I(x, y)$ is slightly blurred with a median filter $M$ on $3 \times 3$ pixel blocks. It avoids possible outliers due to Poisson noise or Moon's small features with relative high intensity differences that can affect the detection of the limb. This type of filtering is more effective than convolution when the goal is to simultaneously reduce noise and preserve edges (Pratt 1978). Each output pixel with coordinates $(x, y)$ contains the median value in the 3-by-3 neighborhood around the corresponding pixel in the input image. Then, an image gradient $G(x, y)$ is processed by convolution with a $3 \times 3$ Prewitt edge detector (Pratt 1978) defined as $P=\left(\begin{array}{rrr}-1 & -1 & -1 \\ 0 & 0 & 0 \\ 1 & 1 & 1\end{array}\right)$, or $-P$ if $y$-axis points to the Moon center. Detection of the limb position in absolute value of the image gradient is determined by a centroid calculation over each column.

We process $N=2000$ images (about one minute of acquisition) that gives a set of limb angular positions obtained at a time t. In order to retrieve transverse AA fluctuations $\alpha_{\perp}$ in a particular set of limb's position, we subtract to this set the temporal mean limb position obtained. The structure function of transverse AA fluctuations is calculated as

$$
D_{\alpha_{\perp}}(\Theta)=\frac{1}{N} \sum_{i=1}^{i=N} \frac{1}{\Theta_{m}-\Theta} \sum_{k=1}^{k=\Theta_{m}-\Theta}\left[\alpha_{\perp}(k)-\alpha_{\perp}(k+\Theta)\right]^{2}
$$

where $\Theta$ is the angular separation in pixels and $\Theta_{m}$ is the maximal extent accessible in the image, i.e. 640 pixels in our case. This differential variance calculated for each image has the practical advantage of being insensitive to vibration effects of the telescope and tracking errors.

The theoretical form of this angular structure function is given by (Borgnino et al. 1992, Avila et al. 1997, Bouzid et al. 2002):

$$
\begin{aligned}
D_{\alpha, t}(\theta)=2.4 \sec & (z) \int_{0}^{+\infty} d h C_{N}^{2}(h) \int_{0}^{+\infty} d f f^{3}\left[f^{2}+\frac{1}{\mathcal{L}_{0}(h)^{2}}\right]^{-11 / 6} \\
& \times\left[1-J_{0}(2 \pi f \theta h)-J_{2}(2 \pi f \theta h)\right]\left[\frac{2 J_{1}(\pi \mathrm{D} f)}{\pi \mathrm{D} f}\right]^{2} \cos ^{2}\left(\pi \lambda h f^{2}\right)
\end{aligned}
$$

where $f$ is the modulus of the spatial frequency, $z$ is the zenithal distance, $\mathrm{D}$ is the aperture diameter, $\mathrm{h}$ is the altitude and $\Theta$ is the angular separation. 

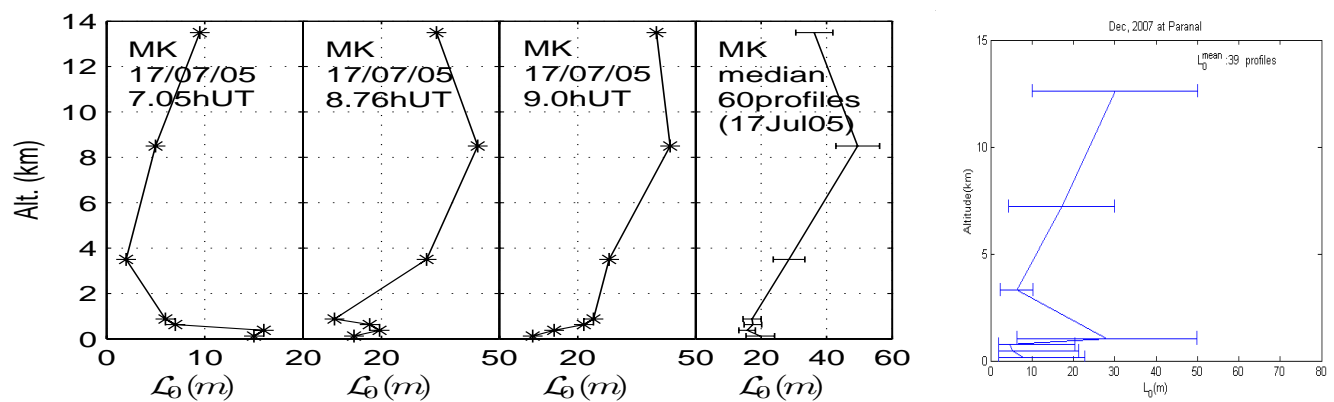

Fig. 2. Examples of outer scale profiles obtained at Mauna Kea and Paranal Observatories during several nights. Altitudes are above the observatory. In the right, median outer scale values for whole campaigns are represented with the standard deviation of the values obtained.

Retrieving $C_{N}^{2}(h)$ and $\mathcal{L}_{0}(h)$ profiles from the AA structure functions (Eq.2) is a non-linear inverse problem. We use simulated annealing (SA) algorithm for minimizing the cost function $E$, defined as the sum over the angular extent of the squared difference between measured and theoretical AA structure functions $E=\sum_{\theta}\left(D_{\alpha_{\perp}}(\theta)-D_{\alpha_{t}}(\theta)\right)^{2}$. This algorithm was developed to statistically find the best global fit of a nonlinear non-convex cost-function (Kirkpatrick et al. 1983).

\section{Results}

Several campaigns have been organized with the MOSP instrument. The first one was performed at the Observatoire de Haute Provence (OHP) in France between the 24th November and 06th December 2004 (Maire et al. 2007). Site testing instruments GSM (Martin et al. 1994, Ziad et al. 2000) and SCIDAR (Fuchs et al. 1998) were also observing simultaneously. During the OHP campaign, a 20-cm telescope (f/15) was used with a $\times 3$ diverging Barlow lens. The Second observation campaign has been carried out at the Mauna Kea Observatory in Hawaii between the 13th and 19th July 2005 with simultaneous observation using the SCIDAR instrument installed at the UH $2.2 \mathrm{~m}$ telescope, $150 \mathrm{~m}$ northeast of the $0.61 \mathrm{~m}$ telescope used by the MOSP. The UH $60 \mathrm{~cm}$ telescope is a Cassegrain with a $9.24 \mathrm{~m}$ focal length (f/15.2). A third campaign was carried out in December 2007 at the Paranal Observatory with other site testing instruments as DIMM, MASS, GSM and SCIDAR. During this campaign a Celestron $28 \mathrm{~cm}$ has been used with a $\mathrm{f} / 10$ focal.

When the difference in altitude between the MOSP and the SCIDAR is significant, a difference of total turbulent energy on the propagation path can occur. Firstly, we estimate both $C_{N}^{2}(h)$ and $\mathcal{L}_{0}(h)$ from the Moon limb. We compensate the value of the SCIDAR $C_{N}^{2}$ in the lower slices until the $C_{N}^{2}$ integration over altitude corresponds to the initial integration of $C_{N}^{2}(h)$ estimated with Moon's limb. Then, these modified simultaneous SCIDAR $C_{N}^{2}$ profiles are used with the SA algorithm in order to have only $\mathcal{L}_{0}(h)$ as unknowns. Total error expected is about $10 \%$. The vertical resolution obtained is a trade-off between the need to have a good sample of the first kilometer above the observatory and the reliability expected for results.

Fig. 2 shows examples of profiles obtained during the Mauna Kea and Paranal campaigns . Median profiles obtained during whole missions show lower values of the outer scale for the first kilometer of the atmosphere. This result can be completed with theoretical relation found by (Ishimaru 1978) who found that the outer scale is on the order of height near the ground.

The simulated annealing algorithm is also appropriate to extract both $C_{N}^{2}(h)$ and $\mathcal{L}_{0}(h)$. In this case, the MOSP can be a completely independent instrument. However, the optimal vertical resolution in the free atmosphere is small compared to the SCIDAR one and results obtained are less reliable. Example of $C_{N}^{2}(h)$ profile obtained is compared with simultaneous Scidar measurement and show a good agreement (Fig. 3).

The comparison between the outer scale measured by GSM and the integrated MOSP outer scale (Borgnino et al. 1990) measured simultaneously gives excellent agreement. 

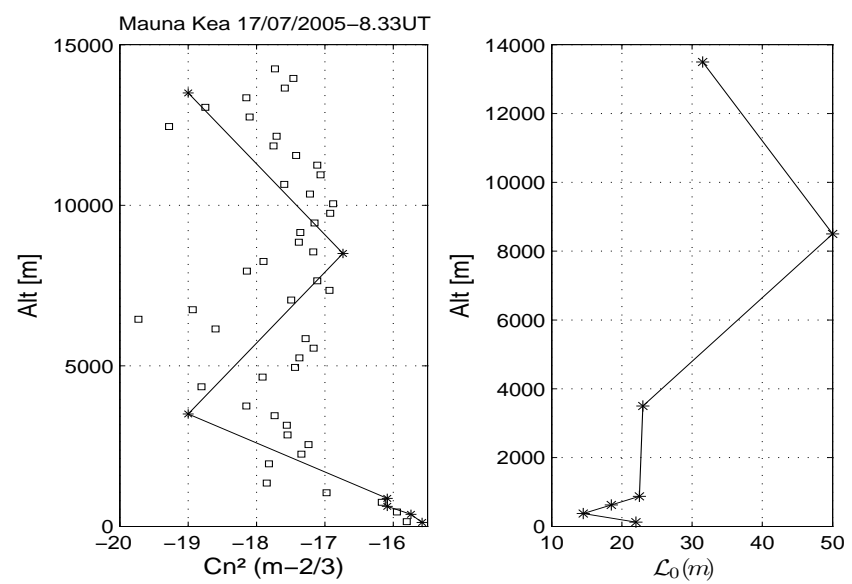

Fig. 3. Example of $C_{N}^{2}$ and outer scale profiles (solid line) that can be retrieved simultaneously using the simulated annealing method. Comparisons with simultaneous SCIDAR profile (square markers) are in close agreement despite different vertical resolutions of these instruments.

\section{Conclusion}

For the first time monitoring of the outer scale profile is possible from Moon's limb observations with a simple device and small telescope apertures. This instrument is also able to extract simultaneously both of $C_{N}^{2}$ and outer scale profiles. From the first observations, measured outer scale profiles exhibit smaller values in the boundary layer than in the free atmosphere. Comparisons with GSM outer scale measurements give excellent agreement.

\section{References}

1. Avila R., Ziad A., Borgnino J., Martin F., Agabi A., Tokovinin A., J. Opt. Soc. Am. A 14, (1997) 3070-3082

2. Borgnino J., Martin F., Ziad A., Optics Communications 91, (1992) 267-279

3. Borgnino J., Appl. Opt. 29, (1990) 1863-1865

4. Bouzid A., Irbah A., Borgnino J., Lantéri, H., Astronomical Site Evaluation in the Visible and Radio

Range (ASP Conf. 266, Ed. Vernin J., Benkhaldoun Z., Muñoz-Tuñón C., 2002), 64

5. Conan R., PhD thesis, Univ. Nice Sophia-Antipolis, 2000

6. Fuchs A., Tallon M., Vernin J., PASP 110, (1998) 86-91

7. Ishimaru A., Wave propagation and scattering in random media II (New York, Academic Press, Inc., 1978)

8. Kirkpatrick S., Gelatt C. D., Vecchi M. P., Science 220, (1983) 671-680

9. Maire J., Ziad A., Borgnino J., Mourard D., Martin F., Jankov S., Bonneau D., Patru F., A\&A 448, (2006) 1225-1234

10. Maire J., Ziad A., Borgnino J., Martin F., MNRAS 377, (2007) 1236-1244

11. Martin F., Tokovinin A., Agabi A., Borgnino J., Ziad A., A\&AS 108, (1994) 173-180

12. Pratt W. K., Digital image processing (A. Wiley-Interscience Publication, New York: Wiley, 1978)

13. Winker D. M., J. Opt. Soc. Am. A 8, (1991) 1568-1573

14. Ziad A., Conan R., Tokovinin A., Martin F., Borgnino J., Appl. Opt. 39, (2000) 5415-5425 